\title{
Evaluation of Wine Colour Under Accelerated and Oak-Cask Ageing Using CIELab and Chemometric Approaches
}

\author{
Maria João Carvalho ${ }^{1,2}$ - Vanda Pereira ${ }^{1,2}$ • Ana C. Pereira ${ }^{1,3}$ • João L. Pinto ${ }^{2}$ • \\ José C. Marques ${ }^{1,2}$
}

Received: 16 December 2014 / Accepted: 13 August 2015 /Published online: 23 August 2015

(C) Springer Science+Business Media New York 2015

\begin{abstract}
The present study aims to analyse the evolution of colour and browning of fortified wines submitted to oak and accelerated ageing induced by temperature, both traditional ageing processes of Madeira wines. These wines were followed during the first 2 years of ageing and were simultaneously compared to aged reference samples, in order to disclose if accelerated ageing favours the wine evolution. The data analysis approaches followed two strategies: the first one was based on the monitoring of wine colour parameters based on CIELab methodology, while the second strategy adopted an untargeted approach, taking advantage of all wine information comprised on the UV-vis spectrum. CIELab results indicated that the greatest variation (up to $92 \%$ ) occurs during the vinification, since it was observed that wines produced from the same grape variety (Tinta Negra) acquired different characteristics already on the vinification processes. All wines tend to approach the reference samples during ageing, with the lowest $\Delta E^{*}$ value obtained for Malvasia submitted to canteiro at 2 years of ageing (3.29 units). Sweet Tinta Negra wines, previously submitted to estufagem, presented the most pronounced colour change associated with ageing. The multivariate principal component analysis (PCA) model was built with aged reference samples to evaluate how young wines acquire the reference wine's characteristics and revealed that
\end{abstract}

Maria João Carvalho

mjcarvalho@uma.pt

1 Centre of Exact Sciences and Engineering, University of Madeira, Campus da Penteada, 9000-390 Funchal, Portugal

2 I3N, University of Aveiro, 3810-193 Aveiro, Portugal

3 CIEPQPF, Department of Chemical Engineering, University of Coimbra, Pólo II - Rua Sílvio Lima, 3030-790 Coimbra, Portugal accelerated ageing increases colour evolution and favours the homogeneity of Malvasia wines.

Keywords Wine $\cdot$ Colour $\cdot$ Browning $\cdot$ Spectrophotometric data $\cdot$ Chemometrics

\section{Introduction}

Important relations between spectral data and oenological features have been established, providing valuable information for quality control practices in wineries (Saurina 2010; Pereira et al. 2011a). The simplest case in routine control in wineries concerns the use of optical density measurements at 420,520 and $620 \mathrm{~nm}$ of visible electromagnetic regions in order to evaluate colour intensity and to correlate with specific compound families or browning development. These parameters only take into account the contribution of three wavelengths for the wine colour definition and do not reflect the overall visual perception of the consumer. To overcome this limitation, application of the CIELab universal colour, which takes into account all visible spectrum information, represented an improvement (Ribéreau-Gayon et al. 2006). More recently, the information on the visible region of the electromagnetic spectrum has been analysed together with the UV spectrum range and great results have been obtained for a comprehensive evaluation of wine colour (Arvanitoyannis et al. 1999; Karbowiak et al. 2010).

The current study focuses on a more extensive study of the chromatic characteristics and the browning development of the Madeira wines, during the first 2 years of ageing, submitted to two different ageing processes. Madeira wines are characterized for their oxidative ageing process where browning assumes a central role, determining its typical colour, which ranges from pale yellow to dark brown, regardless of the grape 
variety (Pereira et al. 2013). Although several studies point out that the browning occurs mainly due to the oxidation of phenolic compounds, since they are one of the most readily oxidized wine constituents (Gómez et al. 1995; LopezToledano et al. 2006; Li et al. 2008; Oliveira et al. 2011; Serra-Cayuela et al. 2013); the phenomenon involves sugars, lipids, amino acids and phenols present in wine ( $\mathrm{Li}$ et al. 2008). Usually, wine browning has been evaluated measuring the browning index (BI), by absorbance reading at a single wavelength, 420 or $440 \mathrm{~nm}$ (Sioumis et al. 2005; SerraCayuela et al. 2013). However, the use of UV-vis data associated with multivariate analysis can better reflect this phenomenon (Gómez et al. 1995; Skouroumounis et al. 2005). Indeed, raw data processing by CIELab and chemometrics have been used by several researchers to estimate the browning development (Skouroumounis et al. 2005; Serratosa et al. 2011; Figueiredo-González et al. 2013).

The Madeira wine-ageing process can be conducted in cellar lofts (canteiro), typically for wines made from white varieties, or can include a previous stage of accelerated ageing, known as estufagem, where the wines are heated, at about $45^{\circ} \mathrm{C}$ for at least 3 months. The estufagem process has already been studied in terms of its effect on some chemical constituents of Madeira wines (Câmara et al. 2003; Câmara et al. 2004; Pereira 2011; Pereira et al. 2011b); however, the evolution of wine colour and related phenomena during both ageing processes still need information. In the present study, wine samples aged through canteiro and estufagem (followed by canteiro) were followed during 2 years. The BI and the chromatic characteristics were evaluated and compared with the aged reference wines in order to understand how the colour develops and to measure the impact of estufagem.

\section{Material and Methods}

\section{Samples}

This study is based on five Vitis vinifera $\mathrm{L}$. white varieties: Malvasia (sweet), Boal (medium sweet), Verdelho (medium dry), Sercial (dry) and Tinta Negra (red variety). Four wines with different sweetness were produced from Tinta Negra: sweet (TNSweet), medium sweet (TNMSweet), medium dry (TNMDry) and dry (TNDry), as depicted on Fig. 1. In order to have representative samples, at least $1000 \mathrm{~kg}$ of each grape variety, with about $18^{\circ}$ Brix, was manually harvested.

The grapes were collected from different locations along the island, in 2011, and then the winemaking process occurred at a local winery, according to common practices. The fermentation process was held without grape solids, commonly known as the bica aberta fermentative process and was conducted at about $25^{\circ} \mathrm{C}$ in stainless steel tanks without adding any commercial yeast. According to the grape variety and desired sweetness, the fermentation was stopped at different fermentation stages, with the addition of natural grape spirit (containing $95 \%(v / v)$ of ethanol), raising the alcohol content up to about $17 \%(v / v)$. The fermentation process was stopped for Malvasia, when the density reached $1046 \mathrm{mg} / \mathrm{L}$, followed by Boal at $1026 \mathrm{mg} / \mathrm{L}$, Verdelho at $1021 \mathrm{mg} / \mathrm{L}$ and Sercial at $1000 \mathrm{mg} / \mathrm{L}$. Concerning Tinta Negra wines, TNSweet was fortified at $1045 \mathrm{mg} / \mathrm{L}$, TNMSweet at $1037 \mathrm{mg} / \mathrm{L}$, TNMDry at $1010 \mathrm{mg} / \mathrm{L}$ and TNDry at $999 \mathrm{mg} / \mathrm{L}$.

After fortification, the wines were clarified and stabilized through bentonite clays and albuminocol gelatins (post-fermentation treatments). Thereafter, all wines were divided and submitted to the common ageing processes: estufagem and canteiro. For the estufagem process, each wine was placed into a special pilot scale system equipped with $200-\mathrm{L}$ stainless steel tanks, fitted with heating coils that allow the circulation of pre-heated tap water and maintained at $45{ }^{\circ} \mathrm{C}$ during 4 months. Then, wines were allowed to undergo natural ageing in oak casks at wine cellar lofts (canteiro). For comparison purposes, about $200 \mathrm{~L}$ of each wine was naturally aged only by the canteiro process. The experiment was followed up to 24 months of ageing.

Samples were collected in triplicate at different stages of the winemaking and ageing process: free run juice $(\mathrm{M})$; wine after post-fermentation treatments (W); wine before ageing (0 month), which was collected 6 months after winemaking, and wines after 1, 2, 3, 4, 6, 12, 18 and 24 months of ageing $(1,2,3,4,6,12,18$ and 24 months). The collected samples were kept at $-20{ }^{\circ} \mathrm{C}$ before analysis.

For comparison purposes, another sample set was collected from aged wines (with an average age of 5 years), considered to have the finest characteristics to be attained in this period, which followed only the canteiro ageing process. These reference samples were supplied by the wine producer and involve 13 Malvasia samples (7 from 2007 and 6 from 2008), 9 Boal samples (5 from 2007 and 4 from 2008), 5 Verdelho samples (3 from 2007 and 2 from 2008), 5 Sercial samples (2 from 2007 and 3 from 2008), 4 TNSweet samples ( 2 from 2007 and 2 from 2008), 2 TNMSweet samples (1 from 2008 and 1 from 2009), 3 TNMDry samples ( 2 from 2007 and 1 from 2008) and 4 TNDry samples (2 from 2007 and 2 from 2008).

\section{Sample Preparation}

About $5 \mathrm{~mL}$ of wine samples was centrifuged on an Eppendorf Centrifuge 5702 (Eppendorf AG, Hamburg, Germany) for $5 \mathrm{~min}$ at $3000 \mathrm{rpm}$. Time and rpm conditions were tested as being the ideal to allow the separation of solid particles. No posterior filtration was required. The supernatant was then collected and ready for analysis. For the collection of the total spectrum (chemometric study), each wine was diluted (1:20) in ultra-pure water. Ultra-pure water was obtained from 
Fig. 1 Schematic description of a winemaking and $\mathbf{b}$ ageing of the studied Madeira wines

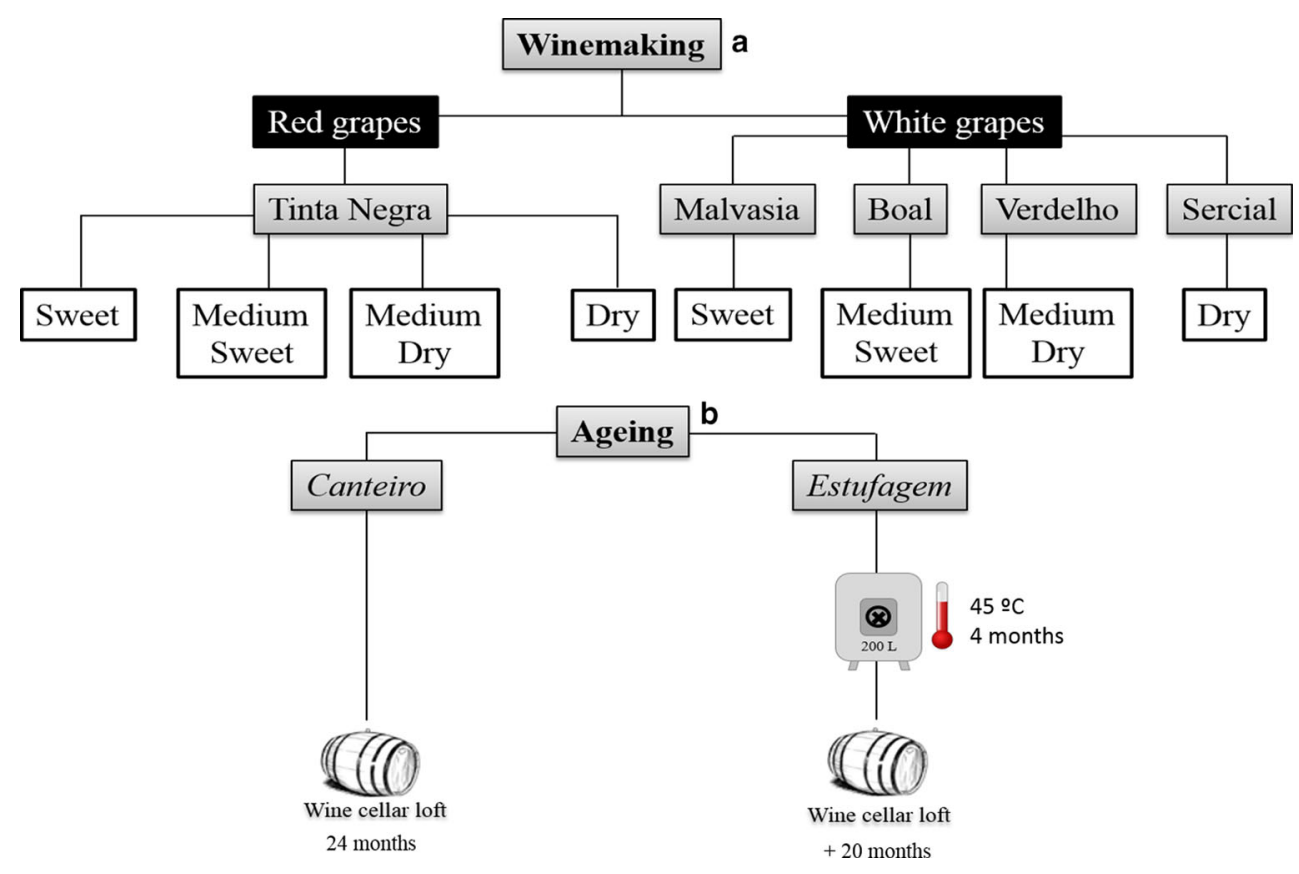

a Millipore Simplicity ${ }^{\circledR}$ UV purification system (Millipore) (Merck Millipore, Darmstadt, Germany) and was always used as blank.

\section{Spectrophotometric Analysis}

Samples were analysed on the dual-beam spectrophotometer Shimadzu UV-vis 2600 (Shimadzu, Kyoto, Japan), using ultra-pure water as blank (OIV 2006). The absorbance readings were performed on quartz cuvettes with an optical thickness of $10 \mathrm{~mm}$. All measurements were carried out in triplicate.

To evaluate the browning development, BI was measured with the absorbance readings at $420 \mathrm{~nm}$.

Since the CIELab system is currently the most used and recommended to evaluate colour, particularly in the food industry (Sant'Anna et al. 2013), and is also an official method of the International Organisation of Vine and Wine (OIV 2006), this methodology was applied in this study. It is a colour scale system based on the opponent-colours theory (HunterLab 2008). CIELab colour or space system is based on a sequential or continuous Cartesian representation of three orthogonal axes: $L^{*}, a^{*}$ and $b^{*}$. Coordinate $L^{*}$ represents clarity ( $L^{*}=0$, black, and $L^{*}=100$, colourless), $a^{*}$ green/red colour component $\left(a^{*}>0\right.$, red, and $a^{*}<0$, green) and $b^{*}$ blue/yellow colour component $\left(b^{*}>0\right.$, yellow, and $b^{*}<0$ blue). Besides these coordinates, the chromatic characteristics of a wine are also defined by its derived magnitudes: chromaticity $\left(C^{*}{ }_{a b}\right)$ and hue $\left(h_{a b}\right)$. The parameters $L^{*}, a^{*}$ and $b^{*}$ were determined measuring the transmittance from 380 to $770 \mathrm{~nm}$ at 5-nm intervals, considering the illuminant D65 (daylight source) and $10^{\circ}$ standard observer, which is currently believed to best represent the average spectral response of human observers (human perception) (HunterLab 2008). In order to study the overall colorimetric difference between two wines, colour differences $\left(\Delta E^{*}\right)$ were calculated as $\Delta E^{*}=\left(\Delta L^{* 2}+\right.$ $\left.\Delta a^{* 2}+\Delta b^{* 2}\right)^{1 / 2}=\left(\left(L_{\text {reference }}^{*}-L_{\text {sample }}^{*}\right)^{2}+\left(a_{\text {reference }}^{*}-a_{\text {sample }}^{*}\right)^{2}+\right.$ $\left.\left(b_{\text {reference }}^{*}-b_{\text {sample }}^{*}\right)^{2}\right)^{1 / 2}$, taking into account the $L^{*} a^{*} b^{*}$ coordinates of the samples and the mean values of the CIELab coordinates of the references (Table 1) (Pérez-Magariño and González-Sanjosé 2003; Sant'Anna et al. 2013). Chromaticity $\left(C^{*}{ }_{a b}\right)$ is considered the quantitative attribute of colourfulness (Gómez-Míguez et al. 2007), corresponding to the intensity of a colour and is calculated as $C_{a b}^{*}=\left(a^{* 2}+b^{* 2}\right)^{1 / 2}$ (Birse 2007). Hue $\left(h_{a b}\right)$ is the attribute that allows to distinguish a colour with reference to a grey colour with the same lightness (Gómez-Míguez et al. 2007), and it is calculated by the

Table 1 Mean values of the CIELab coordinates of each reference

\begin{tabular}{lllllll}
\hline Sample references & $L^{*}$ & SD & \multicolumn{1}{l}{$a^{*}$} & \multicolumn{1}{l}{ SD } & \multicolumn{1}{l}{$b^{*}$} & SD \\
\hline Malvasia & 81.01 & 2.97 & 10.90 & 3.25 & 52.05 & 5.35 \\
Boal & 79.56 & 4.41 & 10.22 & 3.37 & 53.72 & 5.65 \\
Verdelho & 81.51 & 7.66 & 11.16 & 9.87 & 46.83 & 7.88 \\
Sercial & 78.70 & 5.39 & 11.30 & 4.89 & 50.89 & 6.97 \\
TNSweet & 73.69 & 5.12 & 20.20 & 6.52 & 51.33 & 4.23 \\
TNMSweet & 71.00 & 9.65 & 24.03 & 10.42 & 52.15 & 6.37 \\
TNMDry & 65.77 & 9.67 & 29.37 & 6.59 & 54.77 & 1.00 \\
TNDry & 72.96 & 6.31 & 21.05 & 8.14 & 51.87 & 5.43 \\
\hline
\end{tabular}

$S D$ standard deviation 
equation $h_{a b}=\tan ^{-1}\left(\frac{b^{*}}{a^{*}}\right)$ (Pérez-Magariño and GonzálezSanjosé 2003; Birse 2007). For the chemometric study, the absorbance reading was recorded from 190 to $900 \mathrm{~nm}(\Delta \lambda=$ $1 \mathrm{~nm}$ ). Each sample was analysed in triplicate.

\section{Software}

UVProbe 2.42 (Shimadzu, Kyoto, Japan) was used to register the transmittance and absorbance readings. For the automatic calculation of the CIELab parameters $\left(L^{*}, a^{*}, b^{*}\right)$ and $\Delta E^{*}$ values, transmittance data was transferred to Colour Analysis software (vers. 3.10) (Shimadzu, Kyoto, Japan).

\section{Multivariate Analysis-Chemometric Approaches}

The data analysis was performed using principal component analysis (PCA). All data analyses were performed in the computational platform MatLab, with homemade scripts for the PCA model implementation and compute additional tools for the model interpretation (version 7.6, The Mathworks Inc., Natick, MA, USA). Also, data pre-treatment was considered, taking into account data particularities (spectroscopic data usually contain not only chemical but also physical information about samples and measuring conditions). This kind of information is usually irrelevant and can mask the relevant chemical information in the spectra as well as depreciate subsequent analysis (Næs et al. 2002; Rinnan et al. 2009). The most appropriate method for data pre-treatment is highly dependent on the specific application at hand. The variability of the obtained results by different spectral pre-treatment methodologies arises from the fact that individual methods rely on different assumptions about the structure of spectral distortion. In practice, the best spectral pre-treatment is usually obtained upon minimization of the prediction error for a validation set (Faber 1999; Rinnan et al. 2009). In this work, the following pre-processing techniques were analysed and compared: the

\section{Estufagem}

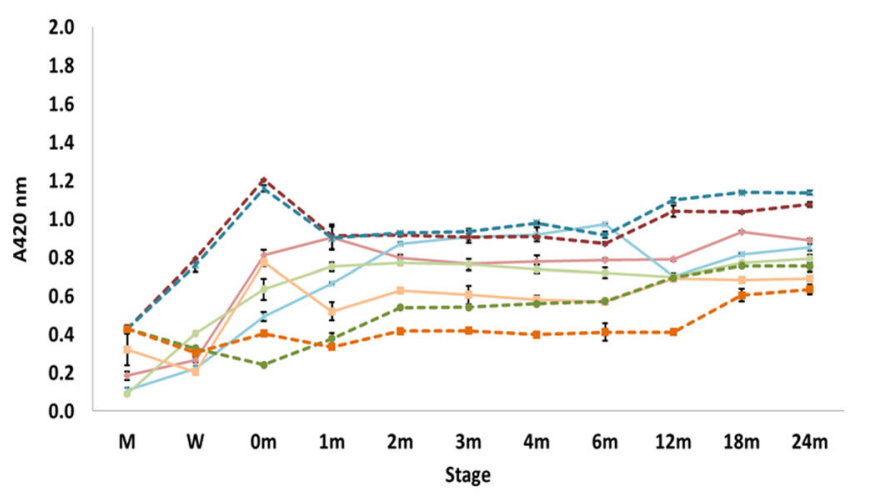

multiplicative scatter correction, MSC, and standard normal variate, SNV. The practical difference between the MSC and SNV approaches is that SNV standardizes each spectrum using only data from that spectrum; that is, it does not use the mean spectrum for all the samples (reference samples), as is the case for MSC. Mathematically, it is identical to performing autoscaling of the rows instead of the columns, i.e., doing autoscaling of the transpose of the data matrix. A detailed description about these techniques can be found elsewhere (Næs et al. 2002). In both cases, absorbance measurements were autoscaled before building the PCA model.

To measure the proximity of the testing samples (those submitted to estufagem and canteiro processes) to the reference samples set, the Hotelling statistics $\left(T^{2}\right)$ was computed. This statistic measures the variability within the PCA subspace, checking whether the projection of a new observation falls inside or outside "normal" conditions (Montgomery 2001). Taking into account that there was a limited number of reference wine samples, it was chosen to perform this analysis only to Malvasia wines, for which a reasonable number of reference samples were available. More samples will be collected for the remaining styles of wines and the approach extrapolated, if this strategy prove to work.

\section{Results and Discussion}

\section{Browning Index}

In terms of BI (Fig. 2), the greatest variations occurred in the first stages of the winemaking (up to 0 month). Browning increase was more relevant for TNSweet and TNMSweet wines, which presented the greatest enhancement in terms of $\mathrm{BI}$, reaching the highest value of about 1.2 AU. According to the pre-established categories defined by Fernández-Zurbano

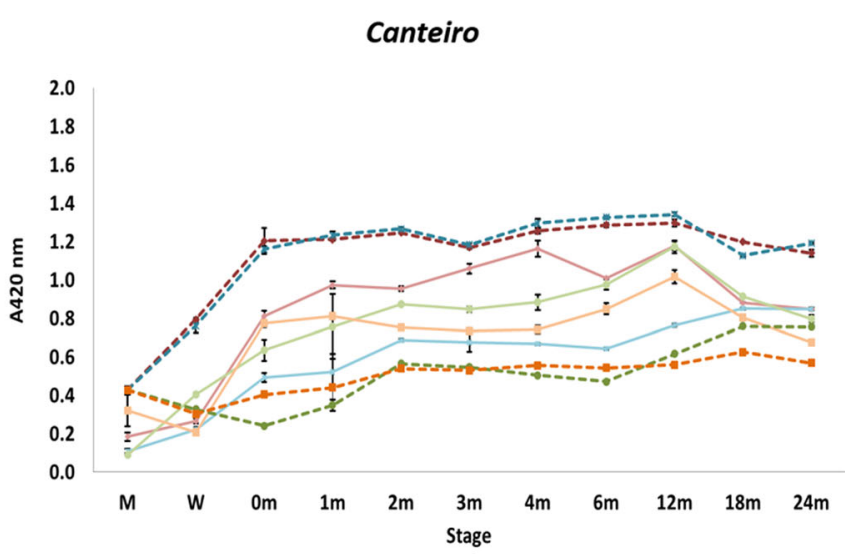
$\longrightarrow$ Malvasia
$-\rightarrow-$ TNSweet $\quad-\cdots-$ TNMSweet $-\bullet-$ TNMDry $\quad--$ TNDry

Fig. $2 \mathrm{~A}_{420}$ measures during winemaking and ageing through estufagem and canteiro 
et al. (1998), this is considered as intense browning $(>0.5 \mathrm{AU})$. This might be explained by some enzymatic browning (flavanol oxidation) especially before the addition of the sulphite solution followed by the non-enzymatic browning that prevails in the wine already fermented ( $\mathrm{Li}$ et al. 2008). The BI for dry Tinta Negra wines showed a slight decrease during fermentation and a slight increase during ageing, indicating a moderate browning (0.2-0.5 AU). In general, all wines presented an increase in the $\mathrm{BI}$, more pronounced in the sweet and medium sweet wines.

The BI of sweet Tinta Negra decreased in the first month of estufagem, stabilizing afterwards. The phenomenon was not observed in canteiro and might be related to the rapid precipitation of high-weight coloured unstable compounds promoted by heating. Indeed, the BI did not present significant changes during the heating step and only a slight increase by the end of the canteiro period was observed probably due to oxidative reactions that can occur in oak casks and clearly detected in the wines aged only by canteiro.

In general, all wines made from white grapes revealed an increase of the BI from vinification up to the end of the 24 months of ageing, regardless of the method of ageing and the sweetness degree. Their final browning can be considered intense (about $0.8 \mathrm{AU}$ ).

These results also revealed that accelerated ageing (estufagem) does not seem to have a considerable impact in the Madeira wine's BI ( $0-4$ months). This can be explained by the fact that during estufagem, the contact of wine with the atmosphere is limited. Mayén et al. (1997) also found that the BI did not increase during the accelerated ageing at $50^{\circ} \mathrm{C}$ of bottled wines made from Pedro Ximenez and Baladi grapes until they were uncorked.

Although the major browning reactions occur through phenolic reactions, it must be stressed that Maillard reactions can also occur, since wine contains the necessary substrates such as amino acids, proteins and reducing sugars. Nevertheless, there is little supportive evidence for its occurrence in wine browning ( $\mathrm{Li}$ et al. 2008) and the present results clearly showed that the greatest increase in absorbance values at $420 \mathrm{~nm}$ occurs before this heating step, with more relevance for sweet and medium sweet Tinta Negra (red variety).

\section{CIELab}

Although $\mathrm{A}_{420}$ indicates the maximum absorbance of the brown pigments (Gómez et al. 1995), there are other important spectrophotometric data for evaluating colour quality.

CIELab results obtained for all samples are presented in Fig. 3. As it can be observed, $L^{*}$ values decreased about 20 units in wines made from white varieties, essentially during vinification, while they barely varied during both ageing processes (0-24 months). At the end, all wines presented similar values, between 84.34 and 86.90 . In turn, $b^{*}$-positive values (yellow) were already found in free run juice of the studied white grape varieties and increased significantly (more than 40 units, about $92 \%$ ) before all wines have been submitted to ageing (before 0 month). The two ageing systems revealed slightly different behaviours for Madeira wines made from white varieties. In general, estufagem promotes a slight decrease of $b^{*}$ values. Then, when wines were submitted to canteiro, significant differences were not observed after 2 years of ageing. Instead, canteiro revealed a slow increase up to 12 months and then a tendency to decrease during the second year of ageing. However, the obtained values at the end of both ageing processes were very close.

In terms of $a^{*}$, the initial coordinates were also positive (red) for the wines made from white grape varieties, except for Verdelho (green hues). This coordinate remained relatively stable during both ageing methods (Fig. 3).

Regarding Tinta Negra wines, there are greater variations in terms of $L^{*}, a^{*}$ and $b^{*}$ parameters when compared to those from white varieties (Fig. 3), and a clear difference was detected between sweet and dry Tinta Negra wines, characteristics already acquired during fermentation. Before ageing, dry wines revealed the tendency to become clearer ( $L^{*}$ increase), while the sweet ones revealed a decrease in this coordinate. However, $L^{*}$ values of these wines increased during ageing, especially during estufagem. Regarding the initial values of $b^{*}$ (positive), there is a tendency to increase before ageing. Initial $a^{*}$ values were higher than $b^{*}$, but with Tinta Negra wine processing, that tendency tends to invert, mostly before ageing (Fig. 3). The $a^{*}$ decrease began during vinification and it was more accentuated for dry wines, remaining almost stable during both ageing processes. In regard to sweet wines, $a^{*}$ continued to decrease especially during estufagem. It is interesting to observe that estufagem seems to accelerate the $a^{*}$ decrease, since the final values are lower for all Tinta Negra styles. The yellow increase $\left(b^{*}\right)$ was more pronounced in sweet than in dry Tinta Negra wines.

It was also found that $L^{*}$ and $a^{*}$ values of sweet Tinta Negra wines aged by both ageing processes evolve with opposite trends, meaning that these wines become clearer with the loss of the reddish tones.

These results can probably mean that the browning phenomenon, promoted by anthocyanin oxidation reactions, is preponderant comparing to parallel reactions that originate high molecular weight polyphenols that precipitate.

Concerning chromaticity (Fig. 4), there is a great difference between dry and sweet Tinta Negra wines, already detected during fermentation, which was not detected in wines made from white varieties. Anthocyanin oxidation products can explain the highest chromaticity presented by sweet Tinta Negra wines. In spite of the ageing process applied, it can be said that Madeira wines tend to similar chromaticity, regardless of their initial colour (Fig. 4). The noticeable difference between sweet and dry wines made from Tinta Negra, which can be 

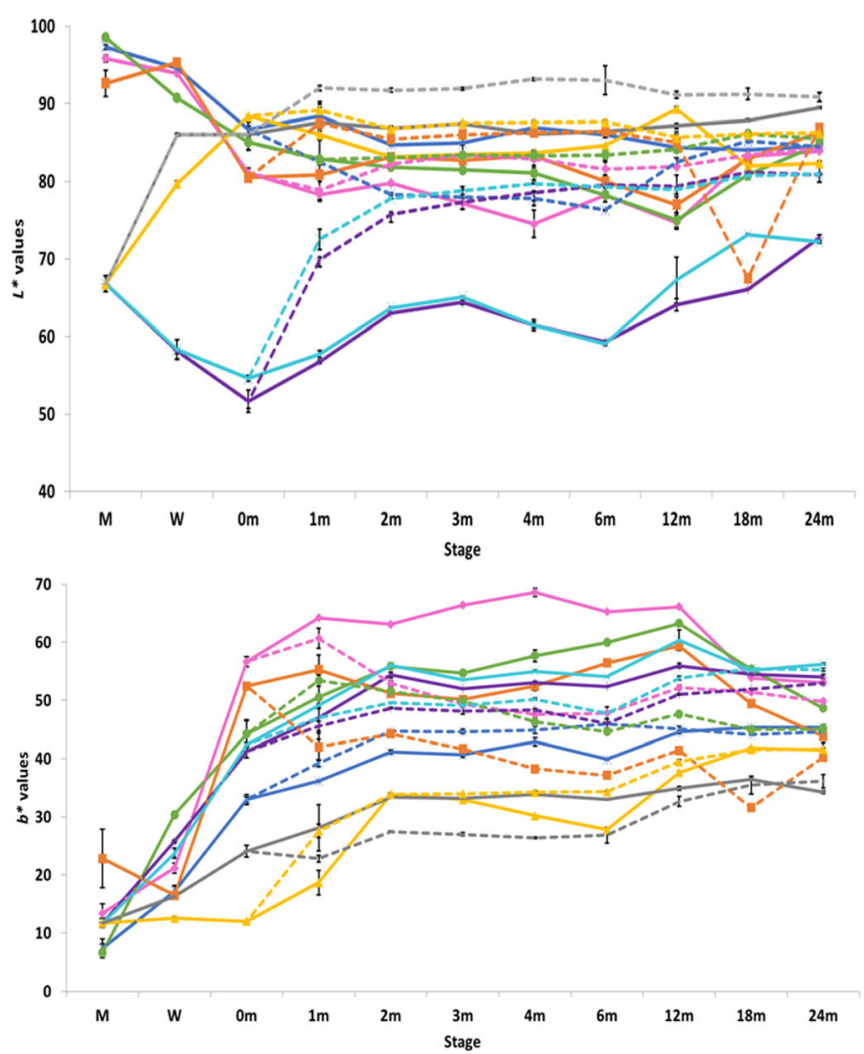

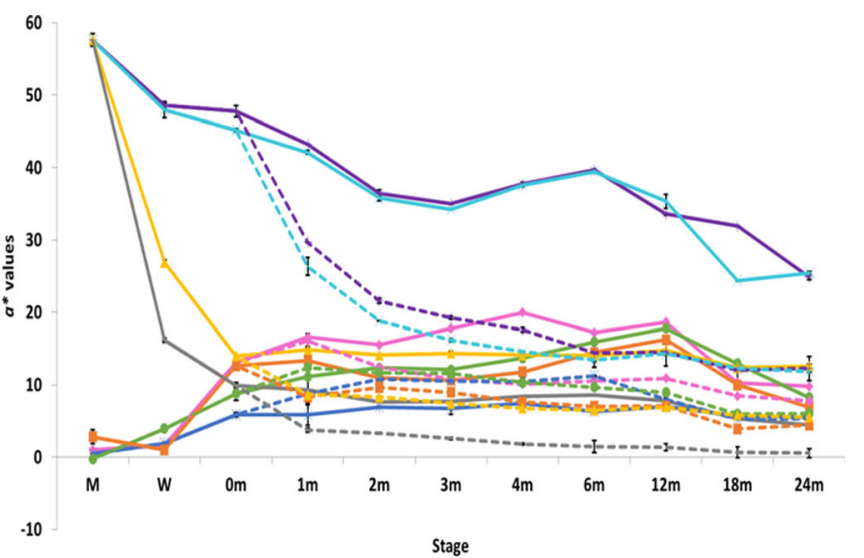

Stage

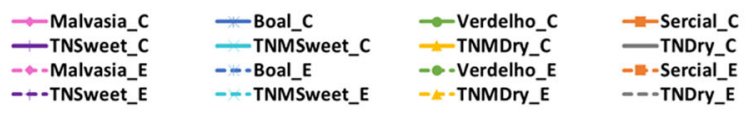

Fig. 3 CIELab coordinates $\left(L^{*} a^{*} b^{*}\right)$ during winemaking and through both ageing processes, canteiro ( $C$ ) and estufagem (E), for wines made from white grape varieties and for wines made from red grape variety

attributed to different extensions of the fermentation period, diminished during both ageing processes. However, the results showed that estufagem has not a clear impact on the chromatic characteristics of all wines.

In terms of hue $\left(h_{a b}\right)$, regarding wines made from white varieties, no variations were observed either in estufagem or in canteiro, as shown in Fig. 4. For Tinta Negra wines, there was a continuous increase of hue before ageing, which then tends to similar values. Nonetheless, there were no significant differences between both ageing processes.

In this sense, the total colour difference $\left(\Delta E^{*}\right)$ is important since it takes into account all differences encountered between $L^{*}, a^{*}$ and $b^{*}$ of the samples and standards, giving us a tool to evaluate the relationship between the visual perception and the numerical analyses (Sant'Anna et al. 2013). To do so, the average of the CIELab results of each sample reference was determined, as described in Table 1. $\Delta E^{*}$ showed a tendency to diminish over time (Fig. 5), indicating that the chromatic characteristics of young Madeira wines tend to approach those of the reference sample set. The $\Delta E^{*}$ results can lead us to think that the same reactions occur in both ageing process. Moreover, the results showed that the estufagem process seems to accelerate the natural colour development of sweet Tinta Negra wines when compared with those aged only by canteiro. This behaviour did not become so evident when analysing the CIELab parameters individually. The approach did not disclose significant differences between wines made from white varieties submitted to both ageing processes.

Generally speaking, the results indicate that there is a tendency to the increase of yellow hues, somehow, traducing the browning development observed in Madeira wines, mostly in the vinification stages. The fact that at the final stage (24 months) the CIElab coordinates, in general, tend to approximate to similar values indicates that estufagem does not seem to have a specific impact on the natural colour development of Madeira wines relative to canteiro.

\section{Chemometrics}

In a previous section, the CIELab data (estimated from the visible region of spectra) was analysed in order to evaluate how colour evolves during both ageing processes (estufagem and canteiro). Nevertheless, it is expected that the ultraviolet region also contains valuable information regarding wine colour changes during ageing.

In consequence, we have used all UV-vis spectra data to analyse how wine evolves during ageing in comparison with the references. With this purpose, a multivariate model was established using wine reference samples, described below. 


\section{Estufagem}
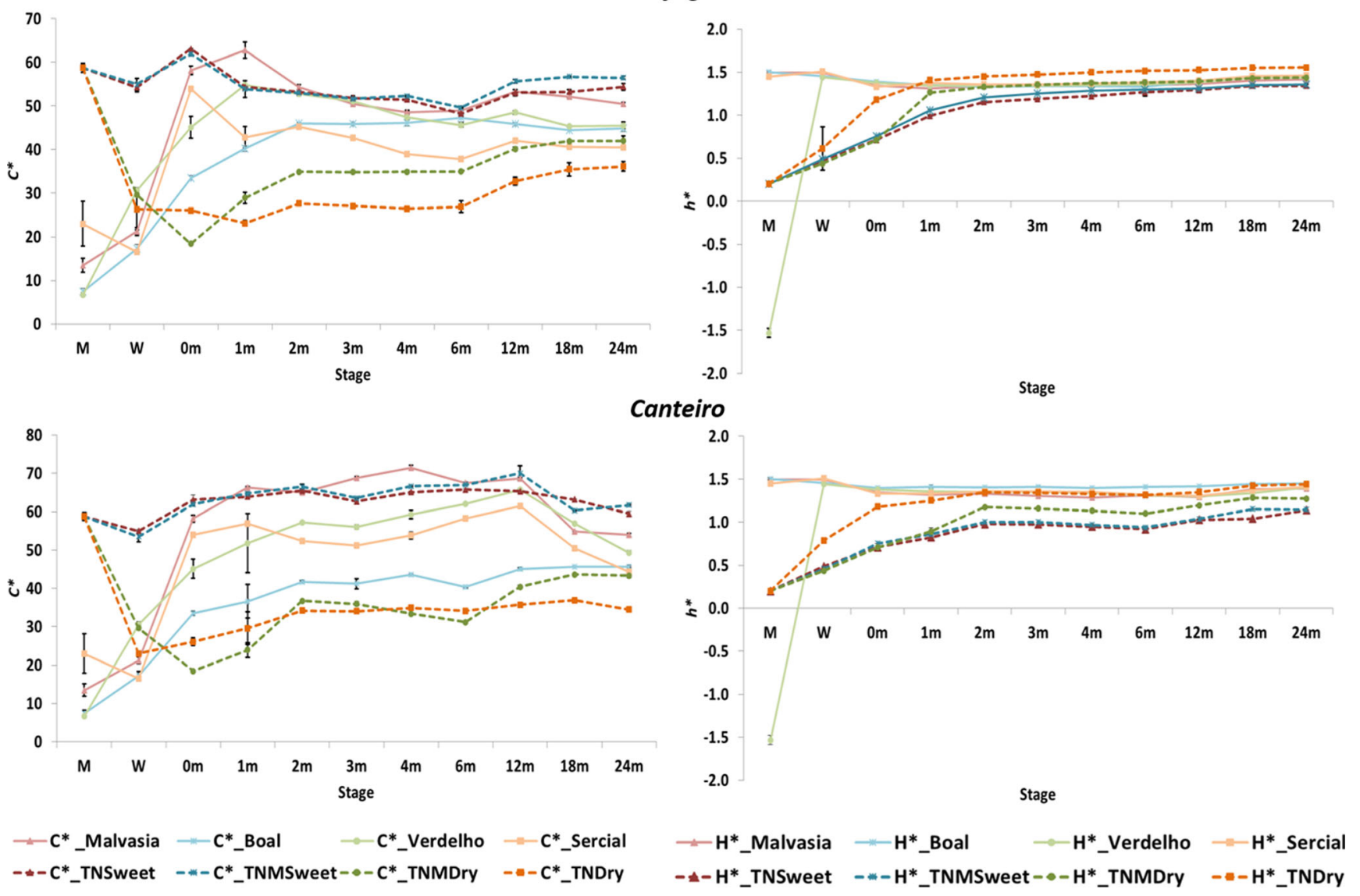

Fig. 4 Chroma $\left(C^{*}{ }_{a b}\right)$ and hue angle $\left(h^{*}{ }_{a b}\right)$ calculated from the CIELab coordinates regarding wines that aged through estufagem and canteiro

A PCA model was established only with Malvasia reference samples to define the typical characteristics of 5-year-old wines. Then, samples collected during the different stages of ageing (from 0 month up to 24 months) were analysed to verify whether their spectral features fall inside the 5-year wine model. Specifically, Hotelling's $T^{2}$ statistic of the PCA scores was evaluated for samples which undergo estufagem and canteiro, to verify whether they became closer to the reference wines. As described in the section "Material and Methods", pre-treatment applied to the spectroscopy data was considered. In this case, the SNV was chosen (leading to higher levels of explained variation) and the PCA model established. The PCA model used the first principal component computed, which explained about $90 \%$ of data variability. The variables with more importance on the first PC are those located in the UV region and vis region up to, approximately, $500 \mathrm{~nm}$ (highest loading values on the first PC). After that, the statistical limits were specified, considering $5 \%$ of significance level, and $T^{2}$ values of the samples during ageing were calculated. The calculated $T^{2}$ values for references and samples are presented in Fig. 6. All reference samples fall inside the confidence limit established. Concerning the remaining samples, it was verified that estufagem samples get closer to the reference samples faster than those of canteiro. At the end of the second month, estufagem samples seem to achieve similar features regarding 5 -year-old reference wines, confirming that indeed estufagem favours the wine evolution in a short period. Moreover, estufagem samples seem to be more similar among them, leading to more homogeneous wine lots.

\section{Conclusions}

For the first time, Madeira wine colour development was deeply studied during 2 years of ageing, evaluating the browning index and the chromatic characteristics achieved through CIELab methodology. The wine samples that aged under two traditional ageing methods, canteiro and estufagem, were compared with the aged reference wines. The CIELab system and chemometric methodologies were successfully applied in order to understand how colour evolves and to disclose if accelerated ageing favours the wine evolution.

The chromatic characteristics evolution of Madeira wines made with white varieties seem to be quite similar regardless of its style (sweetness degree). Concerning Madeira wines 


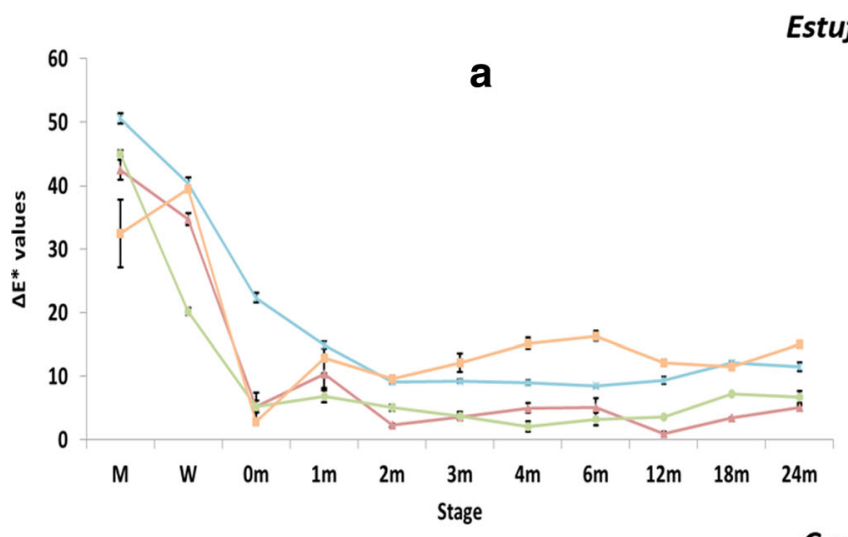

\section{Estufagem}

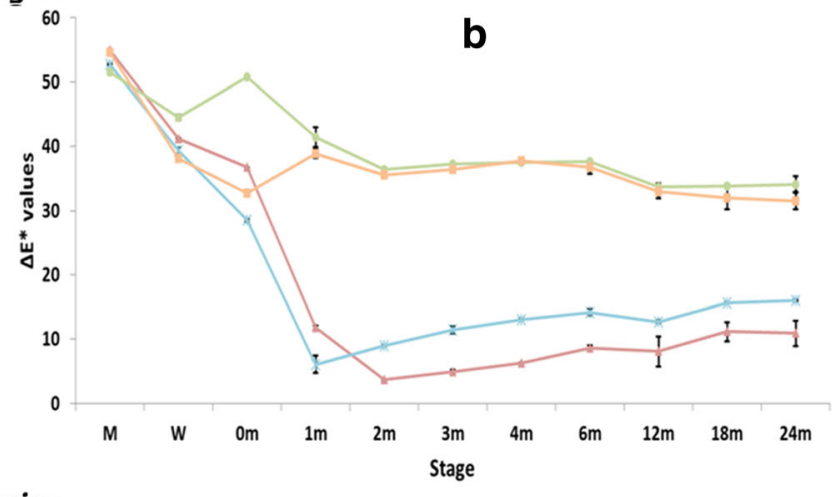

\section{Canteiro}
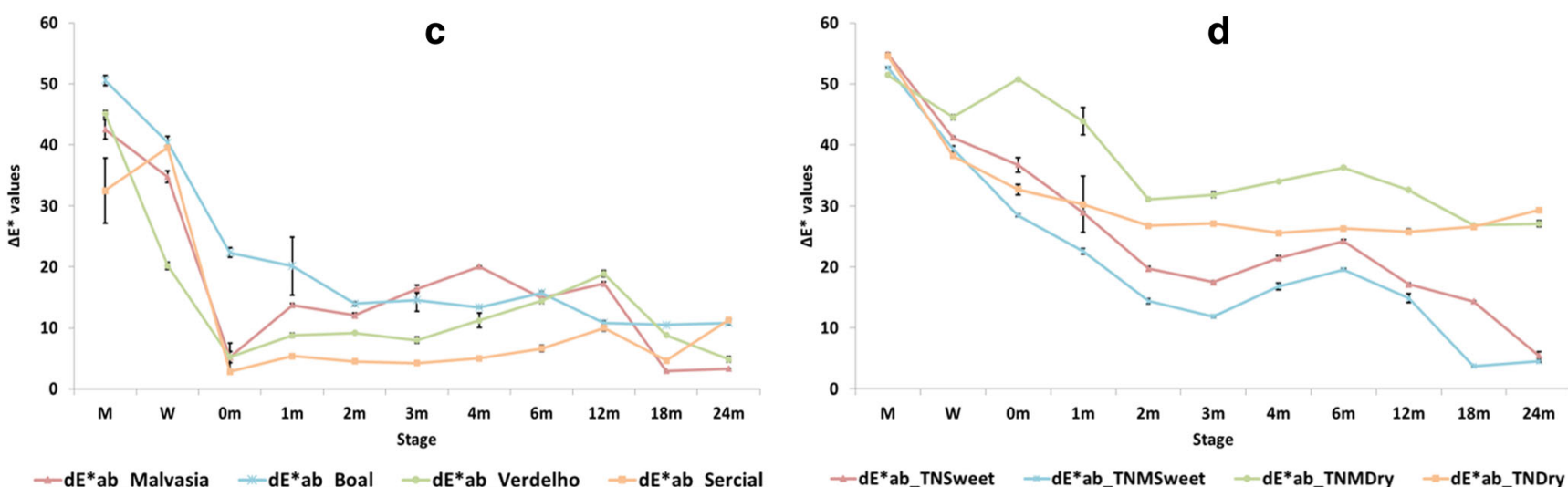

Fig. 5 CIELab parameter $\left(\Delta E^{*}\right)$ showing the tendency of the difference between samples and references, during winemaking and through estufagem and canteiro: $\mathbf{a}$ and $\mathbf{c}$ wines made from white grape varieties; $\mathbf{b}$ and $\mathbf{d}$ wines made from red grape variety

made from Tinta Negra (red variety), there was a clear distinction between sweet and dry wines submitted to estufagem, since the natural browning development, acquired already throughout the vinification procedure, did not reach similar chromatic values, even after ageing. From this study, it can also be concluded that the greatest variations in colour were mostly found in the vinification step (up to $92 \%$ ) and before both ageing procedures were being conducted, even though the following stages seem to be important for the colour stabilization.

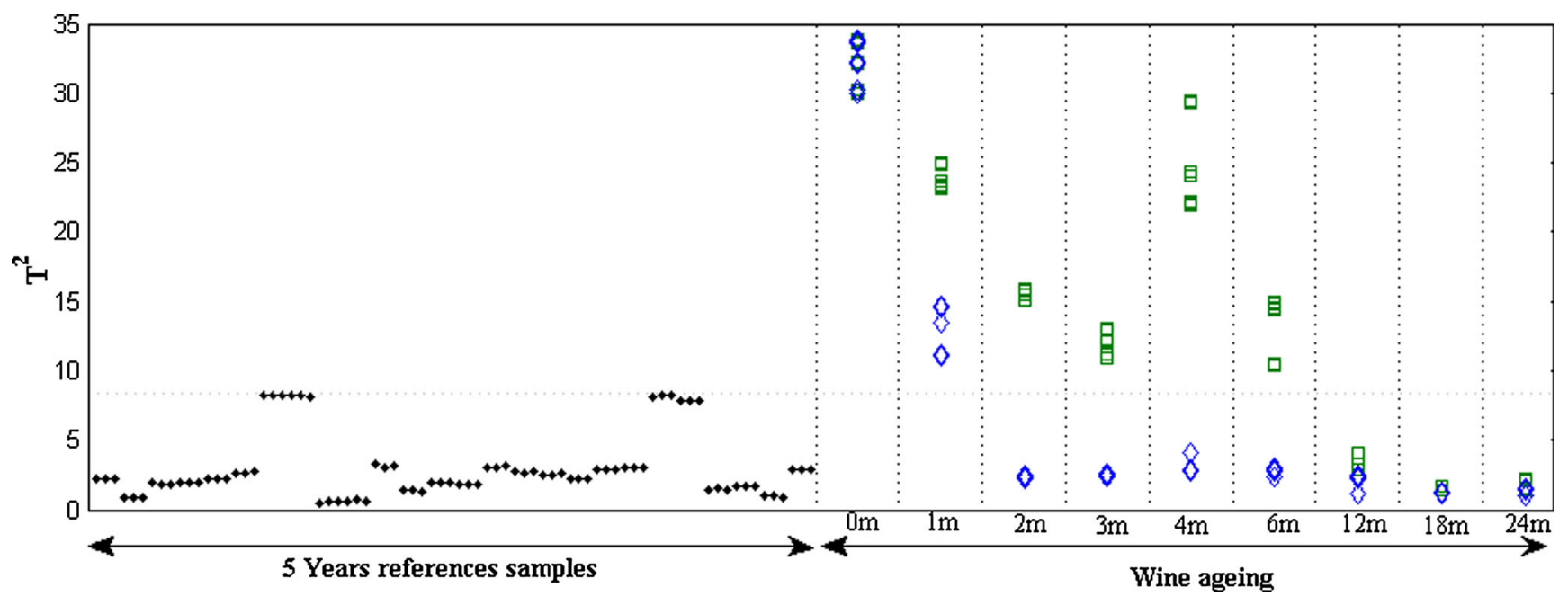

Fig. 6 Plot of $T^{2}$ values of Malvasia reference samples (symbol: asterisk) and of Malvasia samples during estufagem (symbol: rhombus) and canteiro (symbol: square) ageing processes 
The browning index measurements were not as clear as $b^{*}$ values (yellow tone) in order to describe the natural browning development, which was visually detected in all Madeira wines. $b^{*}$ values (yellow tone) of all wines generally increased over time, apparently better reflecting the browning effect. Both ageing processes showed that wine chromatic features evolve in the same trend. However, it was not possible to clearly disclose which ageing procedure hastens the colour development, except for sweet Tinta Negra wines previously submitted to estufagem, as reflected by $\Delta E^{*}$ values. On the other hand, the chemometric study clearly indicates that estufagem ( $45^{\circ} \mathrm{C}$ during 4 months) seems to hasten the acquisition of wine reference characteristics, as reflected by the Malvasia results. Thus, estufagem can be used in all Madeiras, without compromising the typical colour obtained by canteiro. This is an important result since it gives solid bases to wineries as a routine practice. Further studies intend to extend the chemometric approach to the wines made from grape varieties other than Malvasia.

Acknowledgments The authors acknowledge the financial support given by FEDER through + Conhecimento and COMPETE programmes and national funds of FCT (Fundação para a Ciência e Tecnologia): project IMPACT II (MADFDR-01-0190-FEDER-000010), project ESTUFA (PTDC/EQU-EPR/114685/2009, PEst-C/CTM/LA0025/2011) and ARDITI-Agência Regional para o Desenvolvimento da Investigação, Tecnologia e Inovação.

\section{References}

Arvanitoyannis, I. S., Katsota, M. N., Psarra, E. P., Soufleros, E. H., \& Kallithraka, S. (1999). Application of quality control methods for assessing wine authenticity: use of multivariate analysis (chemometrics). Trends in Food Science \& Technology, 10(10), 321-336.

Birse MJ (2007) The colour of red wine. PhD thesis, School of Agriculture, Food \& Wine. Faculty of Science, University of Adelaide, Australia

Câmara, J. S., Marques, J. C., \& Ferreira, A. C. (2003). Heterocyclic acetals in Madeira wines. Analytical and Bioanalytical Chemistry, 375(8), 1221-1224.

Câmara, J. S., Marques, J. C., Alves, M. A., \& Ferreira, A. C. S. (2004). 3-Hydroxy-4,5-dimethyl-2 $(5 \mathrm{H})$-furanone levels in fortified Madeira wines: relationship to sugar content. Journal of Agricultural and Food Chemistry, 52, 6765-6769.

Faber, N. K. M. (1999). Multivariate sensitivity for the interpretation of the effect of spectral pretreatment methods on near-infrared calibration model predictions. Analytical Chemistry, 71(3), 557-565.

Fernández-Zurbano, P., Ferreira, V., Escudero, A., \& Cacho, J. (1998). Role of hydroxycinnamic acids and flavanols in the oxidation and browning of white wines. Journal of Agricultural and Food Chemistry, 46(12), 4937-4944.

Figueiredo-González, M., Cancho-Grande, B., \& Simal-Gándara, J. (2013). Garnacha tintorera-based sweet wines: chromatic properties and global phenolic composition by means of UV-Vis spectrophotometry. Food Chemistry, 140(1-2), 217-224.
Gómez, E., Martínez, A., \& Laencina, J. (1995). Prevention of oxidative browning during wine storage. Food Research International, 28(3), 213-217.

Gómez-Míguez, M., González-Miret, M. L., \& Heredia, F. J. (2007). Evolution of colour and anthocyanin composition of Syrah wines elaborated with pre-fermentative cold maceration. Journal of Food Engineering, 79(1), 271-278.

HunterLab (2008). CIE L*a*b* color scale. Available at http://www. hunterlab.com/appnotes/an07_96a.pdf. Assessed 15 Oct 2014.

Karbowiak, T., Gougeon, R. D., Alinc, J. B., Brachais, L., Debeaufort, F., Voilley, A., \& Chassagne, D. (2010). Wine oxidation and the role of cork. Critical Reviews in Food Science and Nutrition, 50(1), 20-52.

Li, H., Guo, A., \& Wang, H. (2008). Mechanisms of oxidative browning of wine. Food Chemistry, 108(1), 1-13.

Lopez-Toledano, A., Mayen, M., Merida, J., \& Medina, M. (2006). Yeasts used to delay browning in white wines. Food Chemistry, 97(3), 498-504.

Mayén, M., Barón, R., Mérida, J., \& Medina, M. (1997). Changes in phenolic compounds during accelerated browning in white wines from $c v$. Pedro ximenez and $c v$. Baladi grapes. Food Chemistry, 58(I-2), 89-95.

Montgomery, D. (2001). Introduction to statistical quality control. 4 th ed. John Wiley \& Sons Canada, Ltd.

Næs, T., Isaksson, T., Fearn, T., \& Davies, T. (2002). A user-friendly guide to multivariate calibration and classification. Chichester: NIR Publications.

OIV (2006). Détermination des caractéristiques chromatiques selon Cielab (Résolution Oeno 1/2006). In: OIV (ed), vol Méthode OIVMA-AS2-11.

Oliveira, C. M., Ferreira, A. C. S., Freitas, V. D., \& Silva, A. M. S. (2011). Oxidation mechanisms occurring in wines. Food Research International, 44(5), 1115-1126.

Pereira, V. (2011). Effect of the estufagem process on the chemical constituents of Madeira wines. PhD thesis, University of Madeira.

Pereira, A. C., Reis, M. S., Saraiva, P. M., \& Marques, J. C. (2011a). Development of a fast and reliable method for long- and short-term wine age prediction. Talanta, 86, 293-304.

Pereira, V., Albuquerque, F. M., Ferreira, A. C., Cacho, J., \& Marques, J. C. (2011b). Evolution of 5-hydroxymethylfurfural (HMF) and furfural $(\mathrm{F})$ in fortified wines submitted to overheating conditions. Food Research International, 44, 71-76.

Pereira, V., Albuquerque, F., Cacho, J., \& Marques, J. (2013). Polyphenols, antioxidant potential and color of fortified wines during accelerated ageing: the Madeira wine case study. Molecules, 18(3), 2997-3017.

Pérez-Magariño, S., \& González-Sanjosé, M. L. (2003). Application of absorbance values used in wineries for estimating CIELab parameters in red wines. Food Chemistry, 81(2), 301-306.

Ribéreau-Gayon, P., Glories, Y., Maujean, A. \& Dubourdieu, D. (2006). Phenolic compounds. In: Handbook of enology. John Wiley \& Sons, Ltd. pp 141-203.

Rinnan, Å., Berg, F. V. D., \& Engelsen, S. B. (2009). Review of the most common pre-processing techniques for near-infrared spectra. Trends in Analytical Chemistry, 28(10), 1201-1222.

Sant'Anna, V., Gurak, P. D., Ferreira Marczak, L. D., \& Tessaro, I. C. (2013). Tracking bioactive compounds with colour changes in foods - a review. Dyes and Pigments, 98(3), 601-608.

Saurina, J. (2010). Characterization of wines using compositional profiles and chemometrics. TrAC Trends in Analytical Chemistry, 29(3), 234-245.

Serra-Cayuela, A., Aguilera-Curiel, M. A., Riu-Aumatell, M., Buxaderas, S., \& López-Tamames, E. (2013). Browning during biological aging and commercial storage of Cava sparkling wine and the use of 5HMF as a quality marker. Food Research International, 53(1), 226231. 
Serratosa, M. P., Lopez-Toledano, A., Medina, M., \& Merida, J. (2011). Characterisation of the colour fraction of Pedro Ximenez Andalusian sweet wines. South African Journal of Enology and Viticulture, 32(1), 155-163.

Sioumis, N., Kallithraka, S., Tsoutsouras, E., Makris, D. P., \& Kefalas, P. (2005). Browning development in white wines: dependence on compositional parameters and impact on antioxidant characteristics. European Food Research and Technology, 220(3-4), 326-330.
Skouroumounis, G. K., Kwiatkowski, M. J., Francis, I. L., Oakey, H., Capone, D. L., Peng, Z., Duncan, B., Sefton, M. A., \& Waters, E. J. (2005). The influence of ascorbic acid on the composition, colour and flavour properties of a Riesling and a wooded Chardonnay wine during five years' storage. Australian Journal of Grape and Wine Research, 11(3), 355-368. 